\title{
Triangles Generated by Powers of Triplets on the Unit Circle
}

\author{
Charles R. Johnson* and Morris Newman
}

Institute for Basic Standards, National Bureau of Standards, Washington, D.C. 20234

(June 7, 1973)

Let $\alpha, \beta, \gamma$ be three distinct complex numbers of modulus 1 . It is shown that there is essentially one exception to the following statement: For some positive integer $m, 0$ is in the closed convex hull of $\alpha^{m}, \beta^{m}, \gamma^{m}$. The exception occurs for the normalized triple

$$
1, e^{2 \pi i / 7}, e^{2 k \pi i / 7},
$$

where $k=3$ or 5 . This question was motivated by the problem of determining when a positive integer $m$ and a nonzero $n \times 1$ vector $x$ exist such that

$$
x^{*} A^{m} x=0,
$$

where $A$ is a given matrix of $M_{n}(C)$.

Key words: Convex hull; unit circle; Weyl's Theorem.

In connection with determining for what $A \epsilon M_{n}(C)$ the equation

$$
x^{*} A^{m} x=0
$$

is solvable by some positive integer [1] $m$ and some $n \times 1$ vector $x \neq 0$, the following question arose: How many distinct points $\alpha_{1}, \ldots, \alpha_{l}$ on the unit circle are in general required to insure that for some positive integer $m, 0$ lies in the convex hull of $\left\{\alpha_{1}^{m}, \ldots, \alpha_{l}^{m}\right\}$ ? We find that in general $l=4$ such points are required. However, our main result is that under appropriate normalization in the case $l=3$ there is exactly one exceptional set.

Throughout $\alpha, \beta, \gamma$ will denote three distinct points on the unit circle in the complex-plane. We shall denote the triangular solid generated by their $m$ th powers by

$$
T_{m}(\alpha, \beta, \gamma)=\operatorname{Co}\left\{\alpha^{m}, \beta^{m}, \gamma^{m}\right\}
$$

Our goal is to determine for which triples $\alpha, \beta, \gamma$, there is a positive integer $m$ such that

$$
0 \epsilon T_{m}(\alpha, \beta, \gamma)
$$

For this purpose we shall identify two triples $(\alpha, \beta, \gamma)$, and $\left(\alpha^{\prime}, \beta^{\prime}, \gamma^{\prime}\right)$ if one may be obtained from the other via any combination of permutation, reflection, and simultaneous rotation. We shall also identify these two triples if

$$
\left\{T_{m}(\alpha, \beta, \gamma) \mid m \epsilon I^{+}\right\}=\left\{T_{m}\left(\alpha^{\prime}, \beta^{\prime}, \gamma^{\prime}\right) \mid m \epsilon I^{+}\right\} .
$$

AMS Subject Classification: $52-00,15 \mathrm{~A} 24,15-\mathrm{A} 63$

*The work of the first author was done while he was a National Academy of Sciences-National Research Council Postdoctoral Research Associate at the National Bureau of Standards, Washington, D.C. 20234 
Subsequent to this identification there is exactly one exception $(\alpha, \beta, \gamma)$ to the statement:

There is an $m \epsilon I^{+}$such that $0 \epsilon T_{m}(\alpha, \beta, \gamma)$.

With respect to the identification mentioned above, we shall take our triples to be in normalized form:

$$
\alpha=e^{2 \pi i z_{1}}, \beta=e^{2 \pi i z_{2}}, \gamma=1
$$

where $0<z_{1}<z_{2}<1$. Letting $\{r\}$ denote the fractional part of the real number $r$, we shall then say that the positive integer $m$ is a solution to the normalized problem $\left(^{*}\right)$ if

$$
\begin{aligned}
& \text { (i) }\left\{m z_{j_{1}}\right\} \leqslant 1 / 2 \\
& \text { (ii) }\left\{m z_{j_{2}}\right\} \geqslant 1 / 2,
\end{aligned}
$$

and

$$
\text { (iii) }\left\{m z_{j_{2}}\right\}-\left\{m z_{j_{1}}\right\} \leqslant 1 / 2 \text {, }
$$

where $\left(j_{1}, j_{2}\right)=(1,2)$ or $(2,1)$. It is clear that $m$ is a solution to the normalized problem $(*)$ if and only if $0 \epsilon T_{m}(\alpha, \beta, \gamma)$.

Example. If $z_{1}=\frac{1}{7}$ and $z_{2}=\frac{3}{7}$ or $\frac{5}{7}$, then the system $(*)$ is not solvable. Only the values $1 \leqslant m \leqslant 7$ need be considered and it is routine to check that for each of these values at least one of (i), (ii), or (iii) is not satisfied.

Our main result is:

Theorem: Let $\alpha, \beta, \gamma$ be distinct complex numbers such that $|\alpha|=|\beta|=|\gamma|=1$. Then there is a positive integer $\mathrm{m}$ such that $0 \epsilon \mathrm{T}_{\mathrm{m}}(\alpha, \beta, \gamma)$ if and only if the normalized form of $\{\alpha, \beta, \gamma\}$ is not $\left\{\mathrm{e}^{\frac{2 \pi \mathrm{i}}{7}}, \mathrm{e}^{\frac{2 \mathrm{k} \pi \mathrm{i}}{7}}, 1\right\}, \mathrm{k}=3$ or 5 .

Proof: It suffices to consider the normalized problem (*). The necessity then follows from the given example. For the sufficiency we distinguish 5 possibilities (1) $z_{1}$ and $z_{2}$ are irrational and rationally independent; (2) $z_{1}$ and $z_{2}$ are irrational and rationally dependent; (3) exactly one of $z_{1}$ and $z_{2}$ is rational; (4) $z_{1}$ and $z_{2}$ are rational with distinct denominators in reduced form; (5) $z_{1}$ and $z_{2}$ are rational with the same denominator in reduced form.

For cases 1,2, and 3 we shall employ a well-known theorem of Weyl:

LEмma 1 (Weyl [2]): (a) If $z$ is irrational, then the sequence $\{n z\}_{n=1}^{\infty}$ is uniformly distributed on the unit interval. (b) If $z_{1}$ and $z_{2}$ are rationally independent, then the ordered pairs $\left(\left\{n z_{1}\right\},\left\{n z_{2}\right\}\right)$, $n=1,2, \ldots$, are uniformly distributed on the unit square.

In case 1 , the normalized problem (*) is easily solved because of Lemma 1, part (b). For case 2 we assume without loss of generality that the pair $z_{1}, z_{2}$ is of the form

$$
b z, a z+r
$$

where $z$ is irrational, $r=\frac{l_{1}}{l_{2}}$ is rational, and $a, b, l_{1}, l_{2}$ are integers with $a, b, l_{2}>0$. If $a=b$, this case may be transformed into case 3 by rotation. Thus we also assume without loss of generality that $a>b$. Suppose $m=m^{\prime} l_{2}, m^{\prime}>0$ and integral, and let

$$
c=\{m z\}=\left\{m^{\prime}\left(l_{2} z\right)\right\} .
$$


Then by Lemma 1, part (a), we may obtain $c$ arbitrarily close to any real number between 0 and 1 by choice of $m^{\prime}$. Since $a>b>0$, we may choose $\epsilon_{1}, \epsilon_{2}$ so that

and

$$
\epsilon_{1}>0, \epsilon_{2}>0, \epsilon_{1}+\epsilon_{2}<\frac{1}{2}
$$

Next choose

$$
\frac{b}{a}=\frac{1-2 \epsilon_{2}}{1+2 \epsilon_{1}}
$$

$$
0<\epsilon<\min \left\{\frac{1}{2 a}-\frac{\epsilon_{1}}{a}, \frac{\epsilon_{2}}{b}\right\}
$$

Then choose $m^{\prime}$ so that

$$
0 \leqslant c-\left(\frac{1}{2 a}+\frac{\epsilon_{1}}{a}\right)<\epsilon
$$

We then have

$$
\begin{gathered}
\{m(a z+r)\}=\{a c\}=a c \\
\{m(b z)\}=\{b c\}=b c \\
\{m(a z+r)\}-\{m(b z)\}=a c-b c .
\end{gathered}
$$

and

Since

and

$$
\frac{1}{2}+\epsilon_{1} \leqslant a c<\frac{1}{2}+\epsilon_{1}+\epsilon a
$$

it follows that

$$
\begin{aligned}
& \frac{1}{2} \leqslant a c \leqslant 1 \\
& 0 \leqslant b c \leqslant \frac{1}{2}
\end{aligned}
$$

and

$$
0 \leqslant a c-b c \leqslant \frac{1}{2}
$$

so that $(*)$ is solvable in case 2 . In case $3,(*)$ may be solved using Lemma 1 , part (a).

For the remaining cases we assume that in reduced form

$$
z_{1}=\frac{h}{n_{1}}, z_{2}=\frac{k}{n_{2}}
$$

Without loss of generality we may assume that $h$ or $k$ is 1 . If either $n_{i}$ is even, then $0 \epsilon T_{n_{i / 2}}(\alpha, \beta, 1)$ and we are finished. Thus we may assume that $n_{1}$ and $n_{2}$ are odd. Suppose (case 4) that $n_{1} \neq n_{2}$, and $n=$ g.c.d. $\left(n_{1}, n_{2}\right)$. By the Chinese Remainder Theorem, the congruences

$$
2 h m \equiv n_{1}-n \bmod n_{1}
$$


and

$$
2 k m \equiv n_{2}+n \bmod n_{2}
$$

have a solution $m$, which may be taken positive. It then follows that for such an $m$, (i), (ii), and (iii) are satisfied which completes the discussion of this case.

Finally we assume (case 5) that $n_{1}=n_{2}=n$ which is odd. Without loss of generality we take $h=1$ and since $z_{2}$ is in reduced form we have g.c.d. $(k, n)=1$.

LEMma 2: If $n>2,1<k<n$ are integers, $z_{1}=\frac{1}{n}, z_{2}=\frac{k}{n}$, and $(k, n)=1$, then the following are equivalent:

(1) the system $\left(^{*}\right)$ is solvable for $k=x$;

(2) the system $\left(^{*}\right)$ is solvable for $k=x^{\prime}$, where $x x^{\prime} \equiv 1(\bmod n)$; and

(3) the system $\left({ }^{*}\right)$ is solvable for $k \equiv(1-x)(\bmod n)$.

Proof: The equivalence of (1) and (2) follows from the fact

$$
\begin{aligned}
& \left\{T_{m}\left(e^{\frac{2 \pi i}{n}}, e^{\frac{2 \pi i x}{n}}, 1\right) \mid m \epsilon I^{+}\right\} \\
= & \left\{T_{m}\left(e^{\frac{2 \pi i}{n}}, e^{\frac{2 \pi i x^{\prime}}{n}}, 1\right) \mid m \epsilon I^{+}\right\} .
\end{aligned}
$$

The equivalence of (1) and (3) follows from the fact that

$$
e^{\frac{2 \pi i}{n}}\left(e^{\frac{2 \pi i}{n}}, e^{\frac{2 \pi i x}{n}}, 1\right)=\left(1, e^{\frac{2 \pi i(1-x)}{n}}, e^{\frac{2 \pi i}{n}}\right) .
$$

Now if $k$ is even, we may choose $m=\frac{n-1}{2}$ to satisfy $\left(^{*}\right)$ since $n \neq 1$ is odd. Thus we may assume because of Lemma 2 that $k$ and $k^{\prime}$ are both odd, where $1<k^{\prime}<n$ is the unique solution to $k k^{\prime} \equiv 1 \bmod n$. Because of Lemma 2 , we may also assume that

$$
1<k \leqslant \frac{p+1}{2}
$$

However since $\left(\frac{p+1}{2}\right)^{\prime}=2$ which is even, we need only consider

$$
1<k \leqslant \frac{p-1}{2}
$$

We now wish to determine for which $n\left(^{*}\right)$ is solvable under our present assumptions.

Let $m \equiv k^{\prime}\left(\frac{n+j}{2}\right) \bmod n$ where $j$ is odd and $n<k^{\prime} j<2 n$. Then since $k^{\prime}$ is odd, we have $\left\{\frac{m}{n}\right\}=\left\{\frac{1}{2}+\frac{k^{\prime} j}{2 n}\right\}=\left\{1+\frac{k^{\prime} j-n}{2 n}\right\}=\left\{\frac{k^{\prime} j-n}{2 n}\right\}=\frac{k^{\prime} j-n}{2 n}<\frac{n}{2 n}=\frac{1}{2}$ which means that (i) is satisfied. If $l<j<n$ also, then $\left\{\frac{m k}{n}\right\}=\left\{\frac{n+j}{2 n}\right\}=\left\{\frac{1}{2}+\frac{j}{2 n}\right\}=\frac{1}{2}+\frac{j}{2 n}>\frac{1}{2}$ so that (ii) is satisfied. 
Finally $\left\{\frac{m k}{n}\right\}-\left\{\frac{m}{n}\right\}=\frac{1}{2}+\frac{j}{n}-\frac{k^{\prime} j-n}{2 n}=1-\left(\frac{\left(k^{\prime}-1\right) j}{2 n}\right)$ which is less than $\frac{1}{2}$ if $\left(k^{\prime}-1\right) j>n$.

We now have 4 requirements on $j$ for $(*)$ to be solvable. Since $k^{\prime} \neq 1$ is odd they reduce to

and

$$
j \text { odd }
$$

$$
\frac{n}{k^{\prime}-1}<j<\frac{2 n}{k^{\prime}}
$$

Thus if the interval $\left(\frac{n}{k^{\prime}-1}, \frac{2 n}{k^{\prime}}\right)$ is of length greater than or equal to 2 , there will be a solution with $j$ odd and integral. Thus we require

$$
\frac{2 n}{k^{\prime}}-\frac{n}{k^{\prime}-1} \geqslant 2
$$

or

$$
\frac{2\left(k^{\prime}-1\right) n-n k^{\prime}}{k^{\prime}\left(k^{\prime}-1\right)} \geqslant 2
$$

or

$$
n \geqslant 2 \frac{k^{\prime}\left(k^{\prime}-1\right)}{\left(k^{\prime}-2\right)}
$$

As a function of $k^{\prime}, 2 \frac{k^{\prime}\left(k^{\prime}-1\right)}{\left(k^{\prime}-2\right)}$ is increasing for $k^{\prime}>2+\sqrt{2}$. If $k^{\prime}=\frac{n-3}{2}$, our requirement becomes

$$
n \geqslant \frac{(n-3)(n-5)}{(n-7)}
$$

or

$$
n \geqslant 15 \text {. }
$$

It remains to check the cases in which $k^{\prime}=3$ or $\frac{n-1}{2}$ for general odd $n$ and the cases $n=3$, $5,7,9,11,13$. By straightforward computation, the latter yield that $(*)$ is solvable in all cases except that mentioned in the example. In the former case we have that

$$
1-\left(\frac{n-1}{2}\right)^{\prime} \equiv 3 \bmod n
$$

since $n$ is odd. Thus by Lemma 2 it suffices to check $k=3$.

In case $k=3$, it is easily checked that $m=\left[\frac{n}{6}\right]+1$, where $[\cdot]$ denotes the greatest integer function, satisfies $\left(^{*}\right)$ for $n \geqslant 12$. The remaining case $n<12$ have already been checked so that the proof is complete.

COROLLARY: If $1 \geqslant 4$ and $\alpha_{1}, \alpha_{2}, \ldots, \alpha_{1}$ are distinct complex numbers of absolute value 1 , then there is a positive integer $\mathrm{m}$ such that

$$
0 \epsilon \operatorname{Co}\left\{\alpha_{1}^{\mathrm{m}}, \ldots, \alpha_{1}^{\mathrm{m}}\right\} .
$$

Proof: Because of the Theorem, it suffices to check the set of four points $e^{\frac{2 \pi i}{7}}, e^{\frac{6 \pi i}{7}}, e^{\frac{10 \pi i}{7}}, 1$. Since 0 is actually in their convex hull, the result is confirmed.

\section{References}

[1] Johnson, C.R., Powers of Matrices with Positive Definite Real Part, to appear.

[2] Weyl, H. Über die Gleichverteilung von Zahlen mod Eins, Math. Ann. 77 (1916), pp. 313-352.

(Paper 77B3\&4--389) 